\title{
Gas trapping in extremely massive clusters in NGC 1365
}

\author{
Emmanuel Galliano ${ }^{1}$ and Danielle Alloin ${ }^{2}$ \\ ${ }^{1}$ Observatório Nacional, MCT, Rio de Janeiro, Brazil \\ ${ }^{2}$ CEA, Saclay, France
}

\begin{abstract}
Three radio cm sources in the central star-forming kpc of the closeby barred Seyfert galaxy NGC 1365 are observed. The complete dataset includes $V L T$ infrared $J, K, L$ and $N$ images and spectra. The main observed features include (i) a rising dust continuum towards the mid-infrared (MIR), (ii) bright $\mathrm{H}$ emission lines and $\mathrm{PAH}$ (polycyclic aromatic hydrocarbon) features, (iii) a bright MIR [NeII] line, and (iv) no detection of MIR [SIV] or [ArIII] lines. For a typical cluster star-formation history and initial mass function, the [ArIII]/[NeII] and [SIV]/[NeII] ratios exhibit an abrupt decrease of several orders of magnitude at an age of $\sim 7$ Myr. The nondetection of [ArIII] and [SIV] indicates that the clusters are of approximately this age.
\end{abstract}

Keywords. galaxies: starburst, galaxies: star clusters, infrared: galaxies

The full poster (in pdf format) is available at http://www. astro.iag.usp.br/〜iaus266/Posters/pGalliano.pdf. 